\title{
Complete pure vibrational spectrum of HD calculated without the Born-Oppenheimer approximation and including relativistic corrections
}

\author{
Sergiy Bubin, ${ }^{1}$ Monika Stanke, ${ }^{2}$ and Ludwik Adamowicz ${ }^{3,4}$ \\ ${ }^{1}$ Department of Physics and Astronomy, Vanderbilt University, Nashville, Tennessee 37235, USA \\ ${ }^{2}$ Department of Physics, Nicholas Copernicus University, ul. Grudziagdzka 5, PL-87-100 Toruń, Poland \\ ${ }^{3}$ Department of Chemistry, University of Arizona, Tucson, Arizona 85721, USA \\ ${ }^{4}$ Department of Physics, University of Arizona, Tucson, Arizona 85721, USA
}

(Received 24 November 2010; published 29 April 2011)

\begin{abstract}
All 18 bound pure vibrational levels of the HD molecule have been calculated within the framework that does not assume the Born-Oppenheimer (BO) approximation. The nonrelativistic energies of the states have been corrected for the relativistic effects of the order of $\alpha^{2}$ (where $\alpha$ is the fine structure constant), calculated using the perturbation theory with the nonrelativistic non-BO wave functions being the zero-order approximation. The calculations were performed by expanding the non-BO wave functions in terms of one-center explicitly correlated Gaussian functions multiplied by even powers of the internuclear distance and by performing extensive optimization of the Gaussian nonlinear parameters. Up to 10000 basis functions were used for each state.
\end{abstract}

DOI: 10.1103/PhysRevA.83.042520

PACS number(s): 31.15.ac, 31.15.aj, 31.15.xt, 31.15.vn

\section{INTRODUCTION}

The HD molecule is an interesting model system to study, particularly with an approach that does not assume the BornOppenheimer (BO) approximation. Due to the asymmetry of nuclear masses, the electrons approach slightly closer the heavier side of the molecule (the deuteron) than the lighter side (the proton), which leads to a small dipole moment in this system. The charge asymmetry of HD can only be described in quantum-chemical calculations if the $\mathrm{BO}$ approximation is not assumed. In a recent article [1], we used our non-BO approach to study the HD charge asymmetry in all its 18 bound pure vibrational states, and we determined that the asymmetry, as measured by the difference between the average electron-deuteron distance and the electron-proton distance, is the highest for the middle states and lowest for the lowest and the highest states. The HD molecule was also studied in our more recent work [2], which aimed at determination of its fundamental vibrational transition. In those calculations, we used our non-BO approach to determine the nonrelativistic energies of the $v=0$ and $v=1$ states and the first-order perturbation theory with the non-BO wave function as the zeroorder function to determine the leading relativistic corrections to the nonrelativistic energies. In this work, we have extended those calculations to all $18 \mathrm{HD}$ pure vibrational states.

The standard method for calculating vibrational spectra of diatomic molecules is based on the potential energy curve (PEC) determined in electronic structure $\mathrm{BO}$ calculations and corrected for various effects not included in the BO calculations. Such an approach was used, for example, in the recent HD calculations by Pachucki and Komasa (P\&K) [3], where the electronic wave function was obtained variationally as an expansion in terms of Gaussian geminals. Their PEC included adiabatic and nonadiabatic corrections, as well as corrections of the orders of $\alpha^{2}, \alpha^{3}$, and $\alpha^{4}$ (where in atomic units $\alpha=1 / c$; $c$ is the speed of light in vacuum) that account for the relativistic and quantum electrodynamics (QED) effects. They estimated their largest uncertainty per state to be about $0.0036 \mathrm{~cm}^{-1}$. In their article, they present a complete list of all 18 vibrational states and for each state the results include the BO nonrelativistic dissociation energy, the adiabatic correction, the nonadiabatic correction, and the $\alpha^{2}, \alpha^{3}$, and $\alpha^{4}$ corrections to this energy. This data enables a direct comparison with the results generated in the non-BO calculations in the present work.

Upon examining the ratio between the adiabatic and nonadiabatic corrections for different pure vibrational states in the work of Pachucki and Komasa [3], one notices that, while for the lowest two states the former correction is about 10 times larger than the latter, for the higher states the two corrections become comparable in magnitude, and for the highest states the nonadiabatic corrections become larger than the adiabatic one. That indicates that the perturbation series used for accounting for the finite masses of the nuclei does not converge uniformly for all the states, and one may perhaps expect some decrease of the accuracy in accounting for the effects of the finite masses of the nuclei as the level of the vibrational excitation increases. As in our calculations, these effects are explicitly included to infinite order in the non-BO energies, these energies can be used to test the reliability of the perturbation approach used in Ref. [3]. This is one of the aims of the present work.

Another aim is related to the relativistic corrections to the vibrational levels considered here. Those corrections are calculated for each state in this work as expectation values of the appropriate operators representing the leading relativistic effects using the non-BO wave function as the zero-order wave function. As such, the corrections include the so-called recoil effects, which are related to the contributions due to the finite nuclear masses to those corrections. As the recoil effects are not typically included in the standard approach based on the BO approximation, an example of which are calculations by Pachucki and Komasa [3], a comparison of their results with the present results should enable estimation of the magnitude of those effects.

Finally, as will be demonstrated, the present non-BO calculations are well converged at the nonrelativistic level for all 18 pure vibrational states of HD. As such, they provide new standards for energies that are obtained in rigorous variational 
all-particle calculations. This level of convergence required several months of continuous calculations on a large parallel computer system.

As the present calculations are performed without the BO approximation, there is no separation of the vibrational and electronic degrees of freedom. The motions of the electrons and the nuclei are coupled. Due to this coupling, the vibrational quantum number (QN) is, strictly speaking, not a "good" QN (while still being meaningful for unambiguous classification of the states of the system). A good QN (i.e., an exact QN) is the one associated with the total rotation of the system. This allows one to use in non-BO calculations a basis set that describes only the states corresponding to a particular rotational QN (the states with the rotational QN equal to zero) separately from states with other rotational QNs. The different states with the zero rotational QN differ from each other in terms of the number of nodes their wave functions have along the radial coordinates. As the nuclei are much heavier than the electrons, the radial nodes first appear in terms of the coordinate that describes the distance between the nuclei (the deuteron and the proton in HD). One can call them vibrational nodes. Wave functions with nodes in terms of the nucleus-electron and electron-electron coordinates appear at higher energies. Thus, the basis functions used to expand the wave functions of the pure vibrational states of HD in the present calculations need to have a flexible behavior along the internuclear coordinate.

The other features that the basis functions need to be capable of describing are the electron-electron $(e-e)$, electron-nucleus $(e-n)$, and nucleus-nucleus $(n-n)$ correlations. For that the basis functions have to explicitly depend on the $e-e, e-n$, and $n-n$ distances. We should note that the $n-n$ correlation is stronger than the $e-e$ correlation because the nuclei are much heavier and the probability of finding them near each other is much smaller than for electrons.

As we have shown in previous works [4-6], good basis functions for non-BO calculations of rotationless states of a diatomic molecule with only $\sigma$ electrons that possess all the above-mentioned desirable features are explicitly correlated Gaussian (ECG) functions multiplied by powers of the internuclear distance. These functions have been used in our previous calculations of the HD molecule [1,2]. The explicitly correlated Gaussians (also called exponentially correlated Gaussians) have been introduced to quantum mechanical calculations of atomic and molecular electronic structures in the pioneering works of Boys [7] and Singer [8]. Later these functions were applied by several groups, including Adamowicz and Sadlej [9] and Rychlewski and coworkers $[10,11]$, in calculations of the electronic correlation energy in small atomic and molecular systems. A feature that makes the correlated Gaussians suitable for atomic and molecular quantum mechanical calculations is the relative simplicity of the algorithms for calculating the overlap and Hamiltonian matrix elements with those functions.

The nonrelativistic Hamiltonian, called the internal Hamiltonian, used in the non-BO calculations is obtained by separating the motion of the center of mass from the laboratory-frame Hamiltonian. The internal Hamiltonian is isotropic (i.e., rotationally invariant or atom-like) with respect to rotations about the center of the internal coordinate system.
Thus, its eigenfunctions have to transform according to the irreducible representations of the fully symmetric group of rotations. In particular, the ground state or any rotationless state is described with a spherically symmetric wave function. Therefore, the ECGs used in non-BO calculations have to be spherically symmetric functions. Multiplying them by powers of the internuclear distance does not change this symmetry.

We recently developed a procedure for calculating $\alpha^{2}$ relativistic corrections for diatomic systems using non-BO wave functions expanded in terms of ECGs and the first-order perturbation theory [12-15]. These procedures are used in the present work.

We start this article with a brief description of the method we used (a more complete description of the method can be found in our recent reviews [4,5]). We also briefly describe the procedure used to calculate the relativistic corrections. The results obtained in the calculations and their comparison with the experimental results and calculations of others are presented in the last section.

\section{THE METHOD USED IN THE CALCULATIONS}

The internal nonrelativistic Hamiltonian, $\hat{H}_{\text {nonrel }}$, for HD used in the present calculations has the following form:

$$
\begin{aligned}
\hat{H}_{\text {nonrel }}= & -\frac{1}{2}\left(\sum_{i=1}^{3} \frac{1}{\mu_{i}} \nabla_{\mathbf{r}_{i}}^{2}+\sum_{i=1}^{3} \sum_{j \neq i}^{3} \frac{1}{m_{0}} \nabla_{\mathbf{r}_{i}} \cdot \nabla_{\mathbf{r}_{j}}\right) \\
& +\sum_{i=1}^{3} \frac{q_{0} q_{i}}{r_{i}}+\sum_{i<j}^{3} \frac{q_{i} q_{j}}{r_{i j}} .
\end{aligned}
$$

In (1) $q_{0}=q_{1}=1$ are the charges of the nuclei (the proton and the deuteron) and $q_{2}=q_{3}=-1$ are the electron charges; $\mathbf{r}_{i}, i=1,2,3$, are the position vectors of the proton and the two electrons with respect to the deuteron (placed in the center of the internal coordinate system and called the "reference particle"); $r_{i}$ are their lengths; $r_{i j}=\left|\mathbf{r}_{j}-\mathbf{r}_{i}\right|$, $m_{0}=3670.4829654 m_{e}$, and $m_{1}=1836.15267261 m_{e}$ are the masses of the deuteron and the proton, respectively [16]; $m_{2}=$ $m_{3}=m_{e}=1$ are the electron masses; and $\mu_{i}=m_{0} m_{i} /\left(m_{0}+\right.$ $m_{i}$ ) is the reduced mass of particle $i$. As one notices, the Hamiltonian (1) is, as mentioned, isotropic and describes three "pseudoparticles" with charges equal to the charges of the original particles, $q_{1}=1$ and $q_{2}=q_{3}=-1$, moving in the central potential of the charge of the reference particle $\left(q_{0}=1\right)$. This motion is coupled through the Coulomb interactions and through the mass-polarization term, $-\frac{1}{2} \sum_{i=1}^{3} \sum_{j \neq i}^{3} \frac{1}{m_{0}} \nabla_{\mathbf{r}_{i}}$. $\nabla_{\mathbf{r}_{j}}$.

To calculate the relativistic corrections of the order of $\alpha^{2}$, we use the Breit-Pauli Hamiltonian and the first-order perturbation theory. We start with the respective operators representing the mass-velocity (MV), Darwin (D), spin-spin (SS), and orbitorbit $(\mathrm{OO})$ interactions in the laboratory coordinate frame and we transform them to the internal coordinate system of the nine coordinates, $\mathbf{r}_{i}, \quad i=1,2,3$. More details of this transformation for the MV, D, and OO corrections can be found in $[12,13]$. 
For HD the transformed MV, D, SS, and OO operators have the following form:

$$
\begin{gathered}
\hat{H}_{\mathrm{MV}}=-\frac{1}{8}\left[\frac{1}{m_{0}^{3}}\left(\sum_{i=1}^{3} \nabla_{\mathbf{r}_{i}}\right)^{4}+\sum_{i=1}^{3} \frac{1}{m_{i}^{3}} \nabla_{\mathbf{r}_{i}}^{4}\right], \\
\hat{H}_{\mathrm{D}}=-\frac{\pi}{2}\left(\sum_{i=2}^{3} \frac{q_{0} q_{i}}{m_{i}^{2}} \delta^{3}\left(r_{i}\right)+\sum_{i=1}^{2} \sum_{j>i}^{3} \frac{q_{i} q_{j}}{m_{j}^{2}} \delta^{3}\left(r_{i j}\right)\right), \\
\hat{H}_{\mathrm{SS}}=2 \pi \frac{q_{2} q_{3}}{m_{2} m_{3}} \delta^{3}\left(r_{23}\right),
\end{gathered}
$$

$$
\begin{aligned}
\hat{H}_{\mathrm{OO}} & \\
= & -\frac{1}{2} \sum_{i=1}^{3} \sum_{j=1}^{3} \frac{q_{0} q_{j}}{m_{0} m_{j}}\left[\frac{1}{r_{j}} \nabla_{\mathbf{r}_{i}} \cdot \nabla_{\mathbf{r}_{j}}+\frac{1}{r_{j}^{3}} \mathbf{r}_{j} \cdot\left(\mathbf{r}_{j} \cdot \nabla_{\mathbf{r}_{i}}\right) \nabla_{\mathbf{r}_{j}}\right] \\
& +\frac{1}{2} \sum_{i=1}^{3} \sum_{j>i}^{3} \frac{q_{i} q_{j}}{m_{i} m_{j}}\left[\frac{1}{r_{i j}} \nabla_{\mathbf{r}_{i}} \cdot \nabla_{\mathbf{r}_{j}}+\frac{1}{r_{i j}^{3}} \mathbf{r}_{i j} \cdot\left(\mathbf{r}_{i j} \cdot \nabla_{\mathbf{r}_{i}}\right) \nabla_{\mathbf{r}_{j}}\right] .
\end{aligned}
$$

The spin-orbit interaction for HD in the ground electronic state is zero due to the singlet multiplicity of the electronic wave function and the total angular momentum of the states considered in this work being zero. The total relativistic Hamiltonian, $\hat{H}_{\text {rel }}$, is the sum of four contributions: $\hat{H}_{\text {rel }}=$ $\alpha^{2}\left(\hat{H}_{\mathrm{MV}}+\hat{H}_{\mathrm{D}}+\hat{H}_{\mathrm{SS}}+\hat{H}_{\mathrm{OO}}\right)$, and the relativistic correction is calculated for each state as the expectation value of $\hat{H}_{\text {rel }}$ with the non-BO wave function representing the state.

The spatial part of the wave function for each of the eighteen pure vibrational states of HD considered here is expanded in terms of the following ECGs [6]:

$$
\phi_{k}=r_{1}^{m_{k}} \exp \left[-\mathbf{r}^{\prime}\left(A_{k} \otimes I_{3}\right) \mathbf{r}\right],
$$

where the internal coordinate vector is $\mathbf{r}=\left\{\mathbf{r}_{1}^{\prime}, \mathbf{r}_{2}^{\prime}, \mathbf{r}_{3}^{\prime}\right\}^{\prime}$ and ' denotes the vector (matrix) transposition. The functions (6) are symmetrized with respect to the electron labels as required by the singlet multiplicity of the electrons. The symmetrization transforms $\mathbf{r}_{2}$ to $\mathbf{r}_{3}$ and vice versa. Even, non-negative numbers in the 0-250 range are used for the $m_{k}$ powers. This range was found sufficient in our previous works $[4,5]$ to effectively describe the nucleus-nucleus correlation and the vibrational oscillations of the wave functions representing pure vibrational states of small diatomic molecules.

In the optimization of the linear and nonlinear parameters of the ECGs, $\left\{c_{k}\right\},\left\{A_{k}\right\}$, and $\left\{m_{k}\right\}$, for each state in the present work we use the Rayleigh quotient, $E=\min \frac{c^{\prime} H c}{c^{\prime} S c}$, which, subjected to the orthogonality condition for excited states, yields the generalized eigenvalue problem,

$$
\mathrm{Hc}=E \mathrm{Sc} .
$$

Here $\mathrm{H}$ and $\mathrm{S}$ are $K \times K$ symmetric matrices of the Hamiltonian and the overlap in the given basis set, and $\mathrm{C}$ is the vector of linear coefficients of the basis functions. $K$ is the size of the basis set. There are $K$-independent solutions of Eq. (7). The $i$ th eigenvalue, $E_{i}$, always remains an upper bound to the exact energy of the $i$ th state regardless of the choice of the nonlinear parameters. However, in order to approximate the wave functions and the corresponding energies with high accuracy it is necessary to perform an extensive optimization of the nonlinear parameters of the basis functions. This optimization has been the most time consuming part of the present calculations despite the use of the analytic (i.e., not finite-difference) energy gradient determined with respect to these parameters. It should be noted that basis functions (6) have to be square integrable, and this happens if $A_{k}$ matrices are positive definite. Instead of imposing restrictions on the $A_{k}$ matrix elements during the optimization, we use the Cholesky-factored form of $A_{k}$, $A_{k} \equiv L_{k} L_{k}^{\prime}$, where $L_{k}$ is a lower triangular matrix. $A_{k}$ is automatically positive definite for any real values of the $L_{k}$ matrix elements. Thus it is convenient to replace $A_{k}$ with $L_{k}$ and minimize the energy with respect to the latter, because no restrictions need to be imposed on the parameters, which are optimized.

The calculations have been performed independently for each state; i.e., the nonlinear parameters of basis functions were obtained independently for each state. The maximum number of basis functions used for each state was 10000 . To generate this number of functions, the basis set was grown from a small randomly selected set of a few dozen functions using a multistep procedure that involves both a stochastic selection (similar to the one used in Refs. [17,18]) and an optimization employing the analytic energy gradient. The procedure was described in our previous work [2]. When the basis set reached the level of 10000 functions for a particular state, the optimization continued and several one-function-at-a-time optimization cycles were performed to achieve additional lowering of the energy. Due to all this effort, the results shown in the next section are well converged. They represent the lowest values ever obtained in all-particle non-BO variational calculations of the HD molecule.

\section{THE RESULTS}

In Table I, we show how the total nonrelativistic energies and total energies that include the relativistic corrections for some selected states converge with the number of basis functions. As the convergence for the lowest two states was shown in our previous work [2], in the present work we only focus of the states from $v=2$ to $v=17$. The selected states, for which we show the energy convergence in Table I, include the lowest two states in the set (i.e., $v=2$ and $v=3$ ), the two middle states (i.e., $v=8$ and $v=9$ ), and the two highest states (i.e., $v=16$ and $v=17$ ). For each state, the energy values obtained with basis sets ranging in size from 7000 to 10000 in increments of 1000 are shown. As one notices, for all states the convergence of both energies is somewhat better for the lower states than for the higher states. While for the $v=2$ and $v=3$ states nine significant digits are essentially converged, for the highest energy states the number of the converged significant digits is reduced to eight. Also, as expected, additional optimization cycles performed for the basis set of 10000 ECGs lowers the energies of the higher states somewhat more than of the lower states.

The energies for all the states, including the $v=0$ and $v=1$ states computed in our previous work [2], obtained with 
TABLE I. The convergence of the total nonrelativistic non-BO energies $\left(E_{\text {nonrel }}\right)$ and the total energies that include the $\alpha^{2}$ relativistic corrections $\left(E_{\mathrm{rel}}\right)$ with the number of the ECG basis functions. Results for $v=2,3,8,9,16$, and 17 are shown. All values are in hartrees.

\begin{tabular}{|c|c|c|c|c|c|c|}
\hline $\begin{array}{l}E_{\text {nonrel }} \\
\text { No. of ECGs }\end{array}$ & $v=2$ & 3 & 8 & 9 & 16 & 17 \\
\hline 7000 & -1.1331817277 & -1.1182334962 & -1.0551731655 & -1.0449395852 & -1.0006633124 & -0.9996082584 \\
\hline 8000 & -1.1331817296 & -1.1182334992 & -1.0551731841 & -1.0449396035 & -1.0006633663 & -0.9996082788 \\
\hline 9000 & -1.1331817312 & -1.1182335016 & -1.0551731957 & -1.0449396157 & -1.0006633953 & -0.9996082898 \\
\hline 10000 & -1.1331817325 & -1.1182335032 & -1.0551732027 & -1.0449396247 & -1.0006634130 & -0.9996082965 \\
\hline $10000^{\mathrm{a}}$ & -1.1331817331 & -1.1182335038 & -1.0551732051 & -1.0449396277 & -1.0006634237 & -0.9996083005 \\
\hline \multicolumn{7}{|l|}{$E_{\text {rel }}$} \\
\hline No. of ECGs & $v=2$ & 3 & 8 & 9 & 16 & 17 \\
\hline 7000 & -1.1331924396 & -1.1182441535 & -1.0551838526 & -1.0449503613 & -1.0006759718 & -0.9996214937 \\
\hline 8000 & -1.1331924416 & -1.1182441565 & -1.0551838723 & -1.0449503801 & -1.0006760252 & -0.9996215149 \\
\hline 9000 & -1.1331924433 & -1.1182441595 & -1.0551838841 & -1.0449503926 & -1.0006760538 & -0.9996215257 \\
\hline 10000 & -1.1331924448 & -1.1182441614 & -1.0551838922 & -1.0449504018 & -1.0006760720 & -0.9996215321 \\
\hline $10000^{\mathrm{a}}$ & -1.1331924454 & -1.1182441620 & -1.0551838943 & -1.0449504041 & -1.0006760827 & -0.9996215363 \\
\hline
\end{tabular}

${ }^{\mathrm{a}}$ Results obtained by performing several additional optimization cycles.

the basis sets of 10000 ECGs are presented in Table II. For each state, the nonrelativistic non-BO energy and the energy that includes the relativistic corrections are shown. We also included in the table the corresponding energies for the $\mathrm{H}+\mathrm{D}$ atom pair at dissociation. These energies enable calculation of the dissociation energy (DE) of HD and to compare this result with the DE of Pachucki and Komasa (P\&K) [3]. This can be done at the nonrelativistic and relativistic levels of theory. At the nonrelativistic level (which in the calculations of $\mathrm{P} \& \mathrm{~K}$ includes the adiabatic and nonadiabatic corrections), our DE of $36406.5104 \mathrm{~cm}^{-1}$

TABLE II. Total nonrelativistic non-BO energies $\left(E_{\text {nonrel }}\right)$ and total energies that include the $\alpha^{2}$ relativistic corrections for bound pure vibrational states of HD in hartrees. The energies have been obtained with 10000 ECG basis functions. Estimates of the remaining uncertainties of the calculated values are shown in parenthesis. The last line shows the dissociation threshold.

\begin{tabular}{lcc}
\hline \hline$v$ & $E_{\text {nonrel }}$ & $E_{\text {rel }}$ \\
\hline 0 & $-1.1654719220(20)$ & $-1.1654828151(20)$ \\
1 & $-1.1489225895(30)$ & $-1.1489333843(30)$ \\
2 & $-1.1331817331(50)$ & $-1.1331924454(50)$ \\
3 & $-1.1182335038(50)$ & $-1.1182441620(50)$ \\
4 & $-1.1040666452(50)$ & $-1.1040772665(50)$ \\
5 & $-1.0906748634(80)$ & $-1.0906854664(80)$ \\
6 & $-1.0780573245(100)$ & $-1.0780679317(100)$ \\
7 & $-1.0662193300(200)$ & $-1.0662299694(200)$ \\
8 & $-1.0551732051(200)$ & $-1.0551838943(200)$ \\
9 & $-1.0449396277(300)$ & $-1.0449504041(300)$ \\
10 & $-1.0355491788(300)$ & $-1.0355600629(300)$ \\
11 & $-1.0270447026(300)$ & $-1.0270557441(300)$ \\
12 & $-1.0194842295(300)$ & $-1.0194954655(300)$ \\
13 & $-1.0129452625(300)$ & $-1.0129567471(300)$ \\
14 & $-1.0075305468(500)$ & $-1.0075423419(500)$ \\
15 & $-1.0033763150(500)$ & $-1.0033885006(500)$ \\
16 & $-1.0006634237(500)$ & $-1.0006760827(500)$ \\
17 & $-0.9996083005(500)$ & $-0.9996215364(500)$ \\
$\mathrm{H}+\mathrm{D}$ & -0.9995916550 & -0.9996049678 \\
\hline \hline
\end{tabular}

agrees to $0.0004 \mathrm{~cm}^{-1}$ with their result of $36406.5108 \mathrm{~cm}^{-1}$. At the relativistic level, which in both approaches includes the $\alpha^{2}$ correction, our DE of $36405.9794 \mathrm{~cm}^{-1}$ differs from their result of $36405.9809 \mathrm{~cm}^{-1}$ by $-0.0015 \mathrm{~cm}^{-1}$. Part of this difference is the difference of $-0.0004 \mathrm{~cm}^{-1}$ mentioned above, which can be attributed to higher-order nonadiabatic effects. The remaining $-0.0011 \mathrm{~cm}^{-1}$ should provide a rough estimate of the recoil effects.

The energies, which include the $\alpha^{2}$ relativistic corrections presented in Table II, are used to calculate the dissociation energies corresponding to the different vibrational levels. The results are shown in Table III. The dissociation energies also include $\alpha^{3}$ and $\alpha^{4}$ QED corrections. As we have not developed

TABLE III. Dissociation energies of HD calculated using the nonBO nonrelativistic energies and the $\alpha^{2}$ relativistic corrections, both obtained in this work, augmented with the $\alpha^{3}$ and $\alpha^{4}$ corrections taken from the work of Pachucki and Komasa (P\&K) [3]. Our results are compared with the $\mathrm{P} \& \mathrm{~K}$ results. All values are in $\mathrm{cm}^{-1}$.

\begin{tabular}{lrrr}
\hline \hline$v$ & This work & P\&K & Diff. \\
\hline 0 & 36405.7814 & 36405.7828 & -0.0014 \\
1 & 32773.6199 & 32773.6224 & -0.0025 \\
2 & 29318.9008 & 29318.9058 & -0.0050 \\
3 & 26038.1484 & 26038.1525 & -0.0041 \\
4 & 22928.8898 & 22928.8940 & -0.0042 \\
5 & 19989.7441 & 19989.7501 & -0.0060 \\
6 & 17220.5290 & 17220.5367 & -0.0077 \\
7 & 14622.4095 & 14622.4158 & -0.0063 \\
8 & 12198.0883 & 12198.0988 & -0.0105 \\
9 & 9952.1081 & 9952.1180 & -0.0099 \\
10 & 7891.1769 & 7891.1923 & -0.0154 \\
11 & 6024.7045 & 6024.7189 & -0.0144 \\
12 & 4365.4243 & 4365.4408 & -0.0165 \\
13 & 2930.3498 & 2930.3661 & -0.0163 \\
14 & 1742.0329 & 1742.0495 & -0.0166 \\
15 & 830.3773 & 830.4030 & -0.0257 \\
16 & 235.0766 & 235.0929 & -0.0163 \\
17 & 3.6360 & 3.6424 & -0.0064 \\
\hline \hline
\end{tabular}


TABLE IV. Comparison the $\alpha^{2}$ relativistic corrections to the dissociation energies obtained in the present work with the corrections reported by Pachucki and Komasa $(\mathrm{P} \& \mathrm{~K})$ [3]. All values are in $\mathrm{cm}^{-1}$.

\begin{tabular}{lccc}
\hline \hline$v$ & This work & P\&K & Diff. \\
\hline 0 & -0.5311 & -0.5300 & 0.0011 \\
1 & -0.5527 & -0.5509 & 0.0018 \\
2 & -0.5707 & -0.5677 & 0.0030 \\
3 & -0.5826 & -0.5804 & 0.0022 \\
4 & -0.5907 & -0.5887 & 0.0020 \\
5 & -0.5947 & -0.5925 & 0.0022 \\
6 & -0.5938 & -0.5915 & 0.0023 \\
7 & -0.5868 & -0.5853 & 0.0015 \\
8 & -0.5758 & -0.5734 & 0.0024 \\
9 & -0.5567 & -0.5551 & 0.0016 \\
10 & -0.5330 & -0.5297 & 0.0033 \\
11 & -0.4985 & -0.4962 & 0.0023 \\
12 & -0.4561 & -0.4532 & 0.0029 \\
13 & -0.4012 & -0.3991 & 0.0021 \\
14 & -0.3332 & -0.3314 & 0.0018 \\
15 & -0.2477 & -0.2471 & 0.0006 \\
16 & -0.1435 & -0.1420 & 0.0015 \\
17 & -0.0169 & -0.0164 & 0.0005 \\
\hline \hline
\end{tabular}

the capabilities to calculate those QED corrections using our non-BO wave functions yet, the values included in our results in Table III were taken from the P\&K work cited in Ref. [3]. In the table, our dissociation energies are compared with the energies obtained by $\mathrm{P} \& \mathrm{~K}$. Upon examining the values in the table, one notices that the two sets of results agree to about $0.01-0.02 \mathrm{~cm}^{-1}$ with our values being consistently lower. Also, even though the difference oscillates somewhat, it is noticeably smaller for lower $v$ values than for higher values.
Finally, in Table IV we compare our $\alpha^{2}$ relativistic corrections to the dissociation energies with those obtained by $\mathrm{P} \& \mathrm{~K}[3]$ to see if any conclusion can be drawn regarding the magnitude of the recoil effects. As one can see, the results in both columns in Table IV are close but not identical. Our values are consistently more negative than the $P \& K$ values. Even though the differences between the results are close to the uncertainties in either of the two sets of calculations, they should provide a rough estimation of the recoil effects.

\section{SUMMARY}

In this work, we performed calculations of all pure vibrational states of the $\mathrm{HD}$ molecule using the non-BO framework and explicitly correlated Gaussian functions. The non-BO wave functions are used to evaluate the $\alpha^{2}$ relativistic corrections. The results of the calculations are compared with the same kind of results obtained by Pachucki and Komasa [3] with an approach based on solving the vibrational problem with the $\mathrm{H}_{2}$ nonrelativistic potential energy curve corrected for adiabatic, nonadiabatic, relativistic, and QED corrections. While the two methods are fundamentally different, the results concerning the dissociation energies corresponding to different vibrational states are consistent. The only more significant difference, which the comparison reveals, is that our dissociation energies for all states are lower than the P\&K energies by about $0.01-0.02 \mathrm{~cm}^{-1}$.

\section{ACKNOWLEDGMENTS}

This work was supported in part by the National Science Foundation. We also acknowledge partial support of this work by the Polish Ministry of Science and Higher Education (Grant No. N202 041 32/1045). We are grateful to the University of Arizona High Performance Computing Services for providing computer resources for this work.
[1] S. Bubin, F. Leonarski, M. Stanke, and L. Adamowicz, J. Chem. Phys. 130, 124120 (2009).

[2] M. Stanke, S. Bubin, M. Molski, and L. Adamowicz, Phys. Rev. A 79, 032507 (2009).

[3] K. Pachucki and J. Komasa, Phys. Chem. Chem. Phys. 12, 9188 (2010).

[4] M. Cafiero, S. Bubin, and L. Adamowicz, Phys. Chem. Chem. Phys. 5, 1491 (2003).

[5] S. Bubin, M. Cafiero, and L. Adamowicz, Adv. Chem. Phys. 131, 377 (2005).

[6] D. B. Kinghorn and L. Adamowicz, J. Chem. Phys. 110, 7166 (1999).

[7] S. F. Boys, Proc. R. Soc. London A 258, 402 (1960).

[8] J. V. L. Longstaff and K. Singer, Proc. R. Soc. London A 258, 421 (1960).

[9] L. Adamowicz and A. J. Sadlej, J. Chem. Phys. 67, 4298 (1977).
[10] W. Cencek, J. Komasa, and J. Rychlewski, Chem. Phys. Lett. 246, 417 (1995).

[11] J. Rychlewski, Adv. Quantum Chem. 31, 173 (1999).

[12] D. Kędziera, M. Stanke, S. Bubin, M. Barysz, and L. Adamowicz, J. Chem. Phys. 125, 014318 (2006).

[13] M. Stanke, D. Kędziera, S. Bubin, M. Molski, and L. Adamowicz,

[14] D. Kędziera, M. Stanke, S. Bubin, M. Barysz, and L. Adamowicz, J. Chem. Phys. 125, 084303 (2006).

[15] M. Stanke, D. Kędziera, S. Bubin, M. Molski, and L. Adamowicz, J. Chem. Phys. 128, 114313 (2008).

[16] See CODATA 2002 recommended values.

[17] Y. Suzuki and K. Varga, Stochastic Variational Approach to Quantum-Mechanical Few-Body Problems, Lecture Notes in Physics (Springer-Verlag, Berlin, 1998).

[18] K. Varga and Y. Suzuki, Phys. Rev. C 52, 2885 (1995). 